\title{
Excitation and suppression of synchrobetatron resonances in high-intensity hadron linacs
}

\author{
Y. Yamane, H. Okamoto®, ${ }^{*}$ and K. Kojima \\ Graduate School of Advanced Science and Engineering, Hiroshima University, \\ 1-3-1 Kagamiyama, Higashi-Hiroshima 739-8530, Japan
}

(Received 20 April 2021; accepted 26 July 2021; published 16 August 2021)

\begin{abstract}
The space-charge potential can be a source of various resonances in high-intensity hadron bunches traveling in linear accelerators at relatively low energy. Those natural resonances, which may occur even without external driving fields, have to be avoided carefully to minimize undesired beam loss. We perform self-consistent multiparticle simulations systematically to locate major resonance stop bands in the tune diagram, starting from equipartitioned and nonequipartitioned beams. Serious excitation of synchrobetatron resonances is confirmed, depending on the emittance condition at injection. It is demonstrated that a synchrobetatron difference resonance of any order can be suppressed strongly by controlling the ratio of the transverse and longitudinal projected emittances. The equipartitioned linac design is shown to broaden the usable operating area in the tune space, though that has almost nothing to do with thermodynamic effects.
\end{abstract}

DOI: 10.1103/PhysRevAccelBeams.24.084201

\section{INTRODUCTION}

The natural Coulomb interaction among charged particles plays a crucial role in the performance of modern accelerators that transport high-intensity hadron beams $[1,2]$. The space-charge-induced effect is particularly severe in an injector linac operating at relatively low energy. Since the beam is exposed to periodic driving potentials created by focusing magnets and radio-frequency (rf) field, it may be resonantly unstable when the machine working point is not properly chosen. Even if the external driving forces are perfectly linear, a variety of nonlinear resonances arise due to the nonlinear nature of the Coulomb potential [3-10].

In a linac, the bunch length is often comparable to the transverse beam size; the bunch is more or less spherical or ellipsoidal unlike a typical long hadron beam accumulated in the synchrotron that follows. The interplay between the transverse betatron motion and longitudinal synchrotron motion will then become more active, giving rise to possible coupling instabilities. The most powerful and effective approach to such a collective beam-dynamics issue is the use of self-consistent simulation codes [11,12]. We here employ the particle-in-cell (PIC) code "WARP" to explore the space-charge-driven resonance over a wide

\footnotetext{
*Corresponding author. okamoto@sci.hiroshima-u.ac.jp

Published by the American Physical Society under the terms of the Creative Commons Attribution 4.0 International license. Further distribution of this work must maintain attribution to the author(s) and the published article's title, journal citation, and DOI.
}

parameter range, taking the standard drift-tube linac (DTL) structure into account.

The transverse beam focusing is provided by arranging quadrupole magnets along the beam line. The most popular arrangement pattern for a DTL is the so-called "FODO" (Focusing-Drift-Defocusing-Drift) consisting of two quadrupole magnets of opposite polarities [2,13]. In the longitudinal direction, the beam is focused by the rf accelerating field between the drift tubes.

As outlined in Sec. II, the simple sinusoidal focusing potential is taken for this simulation study to model the FODO lattice. The variation of the longitudinal focusing force along the beam orbit is also approximated by the sinusoidal waveform. After defining the root-mean-squared (rms) tune depressions, i.e., a measure of the beam density in phase space, we introduce incoherent and coherent resonance conditions in Sec. III. A brief discussion of the coherent quadrupole-mode stability is made in Sec. IV by solving the three-dimensional (3D) rms envelope equations. We then proceed in Sec. $\mathrm{V}$ to systematic WARP simulations that reveal the existence of diverse coupling and noncoupling resonance bands in the tune diagram. Section VI is devoted to the demonstration of difference-resonance suppression by conditioning the initial beam distribution. The present numerical results, summarized in Sec. VII, will be useful in designing nextgeneration high-intensity linacs as well as in optimizing the operating parameters of existing hadron linacs.

\section{MODEL}

For the sake of simplicity, we only consider the linear external fields indispensable to focus the beam in all 
three dimensions. This allows us to concentrate upon nonlinear effects induced solely by the space-charge potential $\phi_{\mathrm{sc}}$. Nonlinearities originating from the linac structure and mechanical errors unavoidable in reality will enhance a part of the intrinsic space-charge-driven resonances studied here.

The longitudinal linear focusing potential alone never satisfies Maxwell's equations; it is always accompanied by an axisymmetric transverse defocusing term. The betatron motion is thus affected by the gap field, which is known as the rf defocusing (RFD). Leaving dominant linear terms only, we reach the following simplified Hamiltonian for the fundamental study of an intense hadron bunch traveling in a DTL [14]:

$$
\begin{aligned}
H= & \frac{p_{x}^{2}+p_{y}^{2}+p_{z}^{2}}{2}+\frac{K_{\perp}(s)}{2}\left(x^{2}-y^{2}\right) \\
& +\frac{K_{\|}(s)}{2}\left(z^{2}-\frac{x^{2}+y^{2}}{2}\right)+I_{q} \phi_{\mathrm{sc}}(x, y, z ; s),
\end{aligned}
$$

where the independent variable is the path length $s$ along the beam line, $K_{\perp}$ and $K_{\|}$are the $s$-dependent periodic functions determined by the lattice structure, and $I_{q}$ is a constant proportional to the generalized beam perveance. The period of the transverse focusing function $K_{\perp}(s)$ is equal to the length of a unit FODO cell, denoted by $L$. Assuming that all drift tubes contain a quadrupole magnet inside, we have two rf gaps in every transverse focusing period. The period of $K_{\|}(s)$ is then $L / 2$. The beam receives no linear defocusing force in the longitudinal direction unless the principle of alternating phase focusing is applied [15-17].

In the present work, we replace the stepwise functions $K_{\perp}$ and $K_{\|}$by the most dominant sinusoidal harmonics to model the standard FODO lattice. As illustrated in Fig. 1, the wavelength of $K_{\perp}$ is twice longer than that of $K_{\|}$. The longitudinal focusing function $K_{\|}$is always positive. It has been confirmed experimentally and numerically that the sinusoidal lattice has the resonance feature very similar to that of the FODO lattice $[18,19]$. The locations of major stop bands do not essentially change depending on which lattice is chosen.

According to our past experience, the WARP code has a better convergence with the sinusoidal focusing, which is most likely due to the smooth variation in the envelope functions. It is unnecessary in this simple model to adjust the step size of numerical integration at the entrance and exit of each magnet, depending on extra parameters (not very essential to the fundamental feature of resonances) such as the quadrupole occupancy factor. In addition, we are preparing for the novel tabletop experiment of spacecharge effects in short hadron bunches by means of the ion-trap system called "Simulator of Particle Orbit Dynamics" (S-POD) [18-21]. As the S-POD typically employs the sinusoidal rf potential for ion confinement,
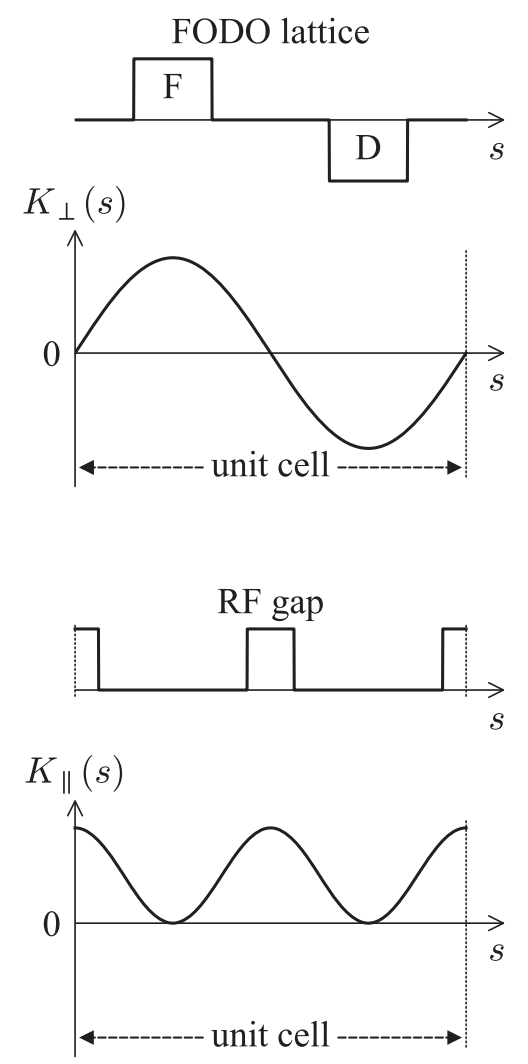

FIG. 1. Sinusoidal focusing model.

the use of the present model is most convenient for us to make a direct comparison of PIC simulation results with forthcoming experimental data.

The rms beam size in $w$-direction is defined by $a_{w}=$ $\sqrt{\left\langle w^{2}\right\rangle}$ where $w$ stands for either $x$ or $y$ or $z$, and the symbol $\left\langle w^{2}\right\rangle$ means taking the average of the quantity $w^{2}$ over the whole phase space. Sacherer proved that $a_{w}$ satisfies the rms envelope equation

$$
\frac{d^{2} a_{w}}{d s^{2}}+\left[K_{w}(s)-\Gamma g_{w}(s)\right] a_{w}-\frac{\varepsilon_{w}^{2}}{a_{w}^{3}}=0,
$$

where $\Gamma$ is the beam perveance, $\varepsilon_{w}$ is the rms emittance given by $\varepsilon_{w}=\sqrt{\left\langle w^{2}\right\rangle\left\langle p_{w}^{2}\right\rangle-\left\langle w p_{w}\right\rangle^{2}}$, and $g_{w}(s)$ depends on the rms beam sizes of the three directions as follows:

$$
g_{w}(s)=\frac{3}{2} \lambda_{3} \int_{0}^{\infty} \frac{d \xi}{\left(a_{w}^{2}+\xi\right) \sqrt{\left(a_{x}^{2}+\xi\right)\left(a_{y}^{2}+\xi\right)\left(a_{z}^{2}+\xi\right)}},
$$

where $\lambda_{3}$ is a constant that takes a value close to $1 / 5 \sqrt{5}$ regardless of the detail of the particle distribution function [22]. The transverse and longitudinal focusing functions are given by $K_{x}=K_{\perp}-K_{\|} / 2, K_{y}=-K_{\perp}-K_{\|} / 2$, and $K_{z}=K_{\|}$according to Eq. (1). The effective phase advance $\mu_{w}$ of $w$-direction, depressed by the space-charge potential, 
can be evaluated by substituting the stationary solution of Eq. (2) in

$$
\mu_{w}=\int_{s}^{s+L} \frac{\varepsilon_{w}}{a_{w}^{2}} d s
$$

$\mu_{w}$ converges to the bare phase advance $\mu_{0 w}$ at the zero intensity where $\Gamma=0$. The rms tune depression of $w$-direction is defined by $\eta_{w}=\mu_{w} / \mu_{0 w}$ that ranges from 0 (high-density limit) to 1 (low-density limit). $\eta_{w}$ is almost independent of what particle distribution the beam possesses in phase space, because of the insensitivity of Eq. (2) to the distribution function.

In the following (except for an example in the Appendix B), we take the symmetric transverse focusing into account, requiring $\mu_{0 x}=\mu_{0 y}\left(\equiv \mu_{0 \perp}\right)$. This condition is naturally fulfilled if the focusing and defocusing quadrupole magnets have an equal field gradient, in other words, the two Q-magnets in a FODO cell are identical. The symmetric focusing is a sort of standard choice in linacs, but in synchrotrons, usually avoided for the reason that the so-called "Montague resonance" may possibly occur at high beam density [23]. Such a coupling resonance in the transverse degrees of freedom can, however, be strongly suppressed by choosing a proper emittance ratio at injection. PIC simulations have shown that no serious difference resonance is activated under the Montague's condition as long as $\varepsilon_{x} / \varepsilon_{y}=1$ [24], which is confirmed even experimentally with the S-POD system [19]. We thus assume $\varepsilon_{x}=\varepsilon_{y}\left(\equiv \varepsilon_{\perp}\right)$ throughout the present study.
Since $\mu_{0 x}=\mu_{0 y}$ and $\varepsilon_{x}=\varepsilon_{y}$, the horizontal and vertical tune depressions are also equal, namely, $\eta_{x}=\eta_{y}\left(\equiv \eta_{\perp}\right)$.

The transverse bare phase advance in the absence of the $\mathrm{rf}$ field $\left(K_{\|}=0\right)$, determined solely by the quadrupole focusing magnets, is always greater than $\mu_{0 \perp}$ that includes the effect of RFD. The quadrupole magnets make no direct effect on the synchrotron oscillation, so the longitudinal bare phase advance $\sigma_{0 \|}$ is simply equal to $\mu_{0 z}$ defined by Eq. (3). We use the symbols $\sigma_{\|}$and $\varepsilon_{\|}$, instead of $\mu_{z}$ and $\varepsilon_{z}$, to express the space-charge-depressed synchrotron phase advance and longitudinal rms emittance.

The matched envelopes $a_{x}, a_{y}$, and $a_{z}$ calculated from Eq. (2) are depicted in Fig. 2. We recognize that the envelope variation is very similar between the sinusoidal lattice and FODO lattice. This is a primary reason why these two types of lattices have almost the same resonance feature.

\section{RESONANCE CONDITIONS}

Before proceeding to numerical simulation data, we propose a simple formula that hopefully predicts the approximate locations of low-order resonance stop bands in the stability map. The Coulomb force generated by each individual particle reaches a long distance. It would, therefore, be quite reasonable to presume that the particles forming the core of an intense beam act in a collective manner.

The beam core has to be defined in the full phase space. In the 3D case of interest to us here, six canonical variables are usually needed to distinguish the core domain.

(a) FODO lattice
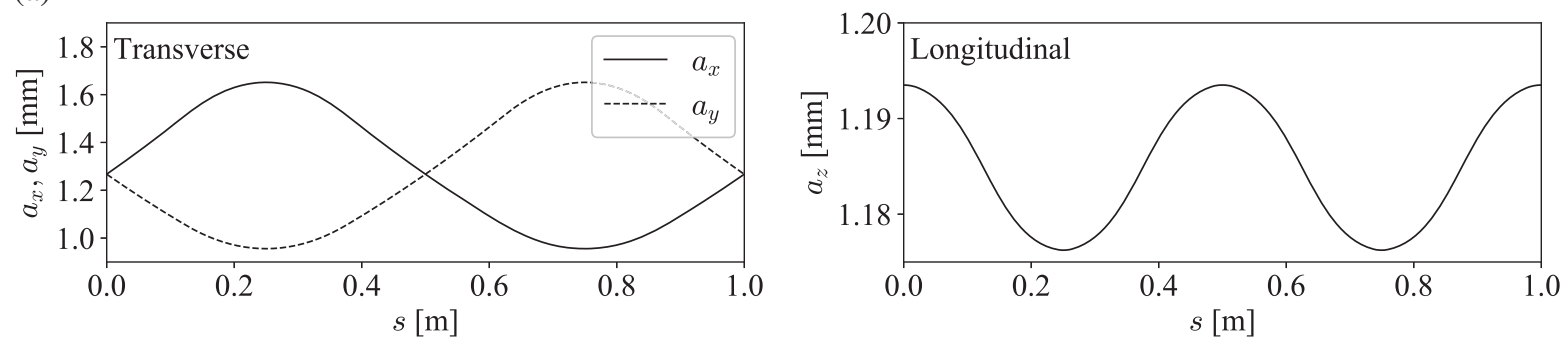

(b) Sinusoidal lattice
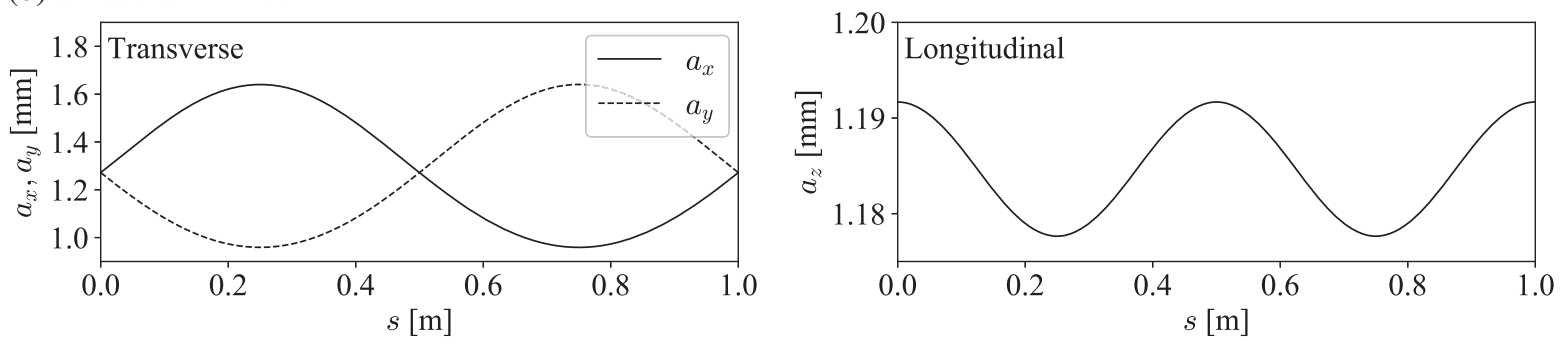

FIG. 2. Transverse and longitudinal rms envelopes matched to (a) the standard FODO lattice, and (b) the sinusoidal lattice in Fig. 1. The operating phase advances are set at $\left(\mu_{0 \perp}, \sigma_{0 \|}\right)=\left(55^{\circ}, 55^{\circ}\right)$ in both cases. As an example, we have assumed an equipartitioned proton beam propagating at the kinetic energy of $10 \mathrm{MeV}$ through a unit cell of $1 \mathrm{~m}$ long. The transverse rms emittance and tune depression are adjusted to $\varepsilon_{\perp}=1.0 \pi \mathrm{mm} \cdot \mathrm{mrad}$ and $\eta_{\perp}=0.7$. 
Particles with relatively high energies in the beam rest frame stay outside the core, forming the tail or, in other words, the halo. These tail particles will act more or less in an incoherent manner because of their weak Coulomb coupling with the core. No general formula is known to define the core boundary as a function of beam density, but the emittance growth of tail origin is separable by checking the total energies of individual particles at injection, see e.g., Ref. [25].

When the space-charge interaction is negligible, each single particle resonates with the alternating gradient (AG) lattice under the condition

$$
n_{\perp} \mu_{0 \perp}+n_{\|} \sigma_{0 \|}=2 \pi n,
$$

where $n_{\perp}, n_{\|}$, and $n$ are all integers. The driving term of this resonance is proportional to $x^{\left|n_{x}\right|} y^{\left|n_{y}\right|} z^{\left|n_{\|}\right|}$where $n_{x}$ and $n_{y}$ are integers related to $n_{\perp}$ as $n_{\perp}=n_{x}+n_{y}$. The resonance order is $m=\left|n_{x}\right|+\left|n_{y}\right|+\left|n_{\|}\right|$.

The single-particle resonance condition in Eq. (4) needs to be modified at high-beam density. For this purpose, the bare phase advances $\left(\mu_{0 \perp}, \sigma_{0 \|}\right)$ in Eq. (4) is often replaced by the space-charge-depressed ones $\left(\mu_{\perp}, \sigma_{\|}\right)$, considering the repulsive effect from the Coulomb potential:

$$
n_{\perp} \mu_{\perp}+n_{\|} \sigma_{\|}=2 \pi n \text {. }
$$

Equation (5) is referred to as the incoherent resonance condition. This type of resonance is expected to take place in the beam tail where individual particles only weakly couple with the core [25-27]. The shifts of the effective phase advances from their design values, i.e., $\Delta \mu_{\perp} \equiv \mu_{0 \perp}-\mu_{\perp}$ and $\Delta \sigma_{\|} \equiv \sigma_{0 \|}-\sigma_{\|}$, are not measurable. They depend on which particle we observe and generally become larger for a particle sitting closer to the center of the core.

The core stability will be affected by some kind of collective mechanism due to the long-range nature of Coulomb interaction. The oscillation amplitudes of particles in the core do not resonantly grow under the incoherent condition in Eq. (5), which has been verified repeatedly by self-consistent theories and numerical simulations, at least in one-dimensional (1D) and twodimensional (2D) cases; linearized Vlasov theories do not predict incoherent instabilities in a matched beam core [3-6,27,28] and PIC simulations also show no significant emittance blowup even when particles deep inside the core satisfy the incoherent resonance condition [25,26,29,30]. The basic mechanisms of resonances in a matched beam could thus be classified into two main categories, namely, the coherent resonance in the core and incoherent resonance in the tail. The coherent core resonance of high order is very weak and believed to be Landau-damped or hardly detectable in practice. Each coherent instability band of low order is accompanied in many cases by the incoherent tail resonances of twice the order. Since the incoherent tune shifts of tail particles are usually not so large as those of core particles, the tail-resonance region lies below the lowtune boundary of the core-resonance band [25-27]. In a strongly mismatched or already collapsed beam, however, a part of tail particles may have large tune shifts in a particular direction. As remarked above, it is vital to pay attention to the particle distribution in six-dimensional full phase space, rather than its projection onto a certain degree of freedom, for the accurate identification of the beam core and tail.

The core motion can be decomposed into collective oscillation modes whose phase advances per cell depend on the beam density. Theoretically, a coherent mode becomes unstable when its oscillation tune is near a half integer [6]. We recently introduced a simple formula to locate the coherent core-resonance bands quickly and easily in betatron tune space [19,25-27]. The proposed 2D resonance condition can be expressed as $n_{x} \mu_{0 x}\left[1-C_{m^{\prime}}(1-\right.$ $\left.\left.\eta_{x}\right)\right]+n_{y} \mu_{0 y}\left[1-C_{m^{\prime}}\left(1-\eta_{y}\right)\right]=\pi n^{\prime}$ where $n^{\prime}$ is an integer, $m^{\prime}=\left|n_{x}\right|+\left|n_{y}\right|$, and $C_{m^{\prime}}$ is a positive constant depending only on the resonance order $m^{\prime}$. The coherent tune-shift factor $C_{m^{\prime}}$ is less than unity but gradually increases for a higher order mode $[6,28,30]$. Notice that, unlike in Eqs. (4) and (5), the right-hand side is $\pi\left(180^{\circ}\right)$ times integer instead of $2 \pi n$. We have confirmed under a variety of initial conditions that, in the beam-density range typical in high-intensity hadron synchrotrons, this simple formula well explains the Vlasov prediction based on the Kapchinsky-Vladimirsky model [27,31-33].

The 2D coherent betatron resonance conjecture above must be generalized to include the synchrotron resonance when the beam is bunched. The most straightforward generalization should be the following:

$n_{\perp} \mu_{0 \perp}\left[1-C_{m}\left(1-\eta_{\perp}\right)\right]+n_{\|} \sigma_{0 \|}\left[1-C_{m}\left(1-\eta_{\|}\right)\right]=\pi n^{\prime}$,

where the symbol $\eta_{\|}$, instead of $\eta_{z}$, is used for the longitudinal rms tune depression. For even $n^{\prime}$, Eq. (6) naturally agrees with the single-particle resonance condition (4) at the low beam-density limit, i.e., $\eta_{\perp}, \eta_{\|} \rightarrow 1$. According to the 1D Vlasov theory by Sacherer, the tuneshift factors of low-order modes are given by $C_{2}=0.750$, $C_{3}=0.875$, and $C_{4}=0.922$ [28]. Similar numbers are reached in another Vlasov theory based on the waterbag model [6]. We have also concluded, through systematic PIC simulations for coasting beams, that $C_{2} \approx 0.7, C_{3} \approx 0.8$, and $C_{4} \approx 0.9$ [26]. These approximate values determined numerically shall be adopted in the following sections.

Equation (6) has been linearized with respect to the space-charge-induced rms tune shifts defined by $\Delta \bar{\mu}_{\perp} \equiv$ $\left(1-\eta_{\perp}\right) \mu_{0 \perp}$ and $\Delta \bar{\sigma}_{\|} \equiv\left(1-\eta_{\|}\right) \sigma_{0 \|}$, just like the previous 
2D resonance conjecture. This linearization impairs the accuracy of locating resonance bands because the phasespace density of a hadron bunch in a linear machine can be much higher than that in a circular machine. The concise resonance criterion as in Eq. (6) will nonetheless help to make a crude but quick estimate of dangerous parameter ranges where the possible beam-quality deterioration or even serious particle losses may occur in linacs.

\section{RMS ENVELOPE ANALYSIS}

Provided that the transverse density profile of a coasting beam has elliptical symmetry, the 2D envelope equations become totally independent of how the particles are distributed in four-dimensional phase space [22]. Even for a bunched beam, if its density has ellipsoidal symmetry in real space, the 3D envelope equations depend only very weakly on the phase-space distribution function. The envelope equations could, therefore, be employed to examine the stability of the linear $(m=2)$ coherent modes that actually possess such symmetry.

The stability of the stationary (matched) solution to the 2D envelope equations against perturbation was analyzed in detail by Struckmeier and Reiser [34]. Their perturbative approach was recently applied by Qiang to the 3D envelope equations in order to explore the bunched-beam stability [35]. We here follow his analysis to figure out the region of the linear-mode instability in the so-called "tune diagram." A couple of typical diagrams on the basis of the sinusoidal focusing model are presented in Fig. 3. Equation (6) predicts a transverse noncoupling resonance and a synchrobetatron resonance along solid lines. The former resonance near the betatron phase advance of $90^{\circ}$ per cell is sometimes referred to as the "envelope instability" studied previously by many researchers [1,4,34,36-39]. A detailed discussion about the latter type of sum resonance can be found in Ref. [40]. Note that the envelope instability band in the constant-intensity case of Fig. 3(b) is slightly curved because the transverse tune depression varies depending on the operating point.

The envelope equations cannot describe the effect of the skew driving term, which is why the linear difference resonance along $\mu_{0 \perp}-\sigma_{0 \|} \approx 0$ is missing in Fig. 3 [25]. The sum resonance observed here is a sort of confluent instability that should be very weak and thus probably causes no serious effect in reality. In PIC simulations, this line appears much more clearly due to the existence of the linear coupling terms proportional to $x z$ and $y z$. Even the linear difference resonance is excited when there exists an emittance imbalance between the transverse and longitudinal directions.

It is worth noting that no synchrotron resonance is activated near $\sigma_{0 \|}=90^{\circ}$. Even when $\sigma_{0 \|}$ is as large as $\mu_{0 \perp}$, the longitudinal breathing motion turns out to be almost negligible as is evident from the example in Fig. 2. Another point, presumably more essential, is that the longitudinal envelope modulation within the first half of the unit cell looks identical to that within the latter half. The synchrotron phase advance per actual oscillation period is, therefore, a half of $\sigma_{0 \|}$. These facts will explain the weakness of the pure synchrotron resonance corresponding to the $n_{\perp}=0$ case in Eq. (6).

\section{SELF-CONSISTENT MULTIPARTICLE SIMULATIONS}

A detailed numerical study beyond the envelope analysis was carried out by the help of the WARP code that enables

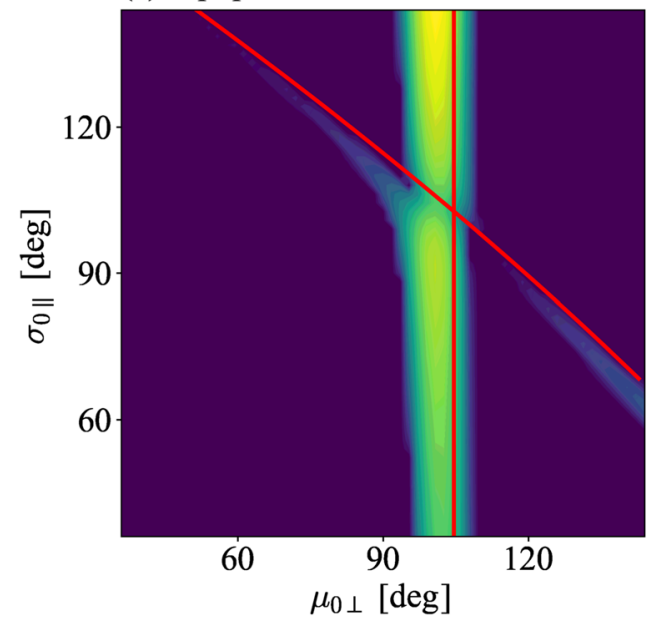

(b) Constant intensity

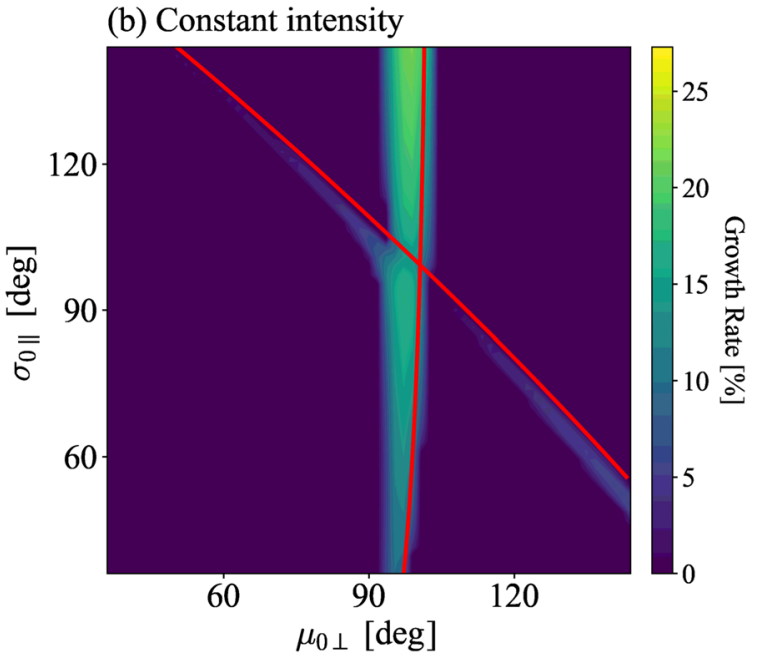

FIG. 3. Linear-mode stability predicted by the 3D rms envelope equations. Red lines indicate the positions of the second-order $(m=2)$ resonances expected from Eq. (6) with $C_{2}=0.7$. In the left panel (a), the equipartitioning condition is applied over the whole tune space with the transverse tune depression $\eta_{\perp}$ fixed at 0.8 . In the right panel (b), the beam intensity (perveance) is kept unchanged. $\eta_{\perp}$ thus depends on the operating point. The intensity is determined so as to achieve $\eta_{\perp}=0.8$ at $\left(\mu_{0 \perp}, \sigma_{0 \|}\right)=\left(55^{\circ}, 55^{\circ}\right)$. 
us to make a self-consistent exploration of bunched-beam stability [12]. We again took the sinusoidal focusing model introduced in Sec. II. The beam transport channel considered here is 100 AG cells long. The number of total sinusoidal periods in the longitudinal direction is doubled as each AG cell has two rf gaps. The initial particle distribution is always matched to the periodic lattice, including the Debye screening effect from the space-charge potential. Such a fine-grained matching procedure is strongly demanded at high-beam density to avoid mismatch-induced emittance growth that obscures the signatures of weak nonlinear resonances. The importance of the self-consistent matching is illustrated in Appendix A. Additional information about the case of asymmetric transverse focusing $\left(\mu_{0 x} \neq \mu_{0 y}\right)$ is also given in Appendix $\mathrm{B}$.

\section{A. Stop bands in initially equipartitioned beams}

Let us start from the case where the initial beam parameters satisfy the so-called "equipartitioning condition" [41]:

$$
\frac{\varepsilon_{\|}}{\varepsilon_{\perp}}=\frac{\eta_{\perp} \mu_{0 \perp}}{\eta_{\|} \sigma_{0 \|}} .
$$

This condition is thought to broaden the stable operating range of a linac while, from a thermodynamic point of view, the energy equipartitioning should develop much more slowly than the timescale considered in our WARP simulations here. The DTL at the Japan Proton Accelerator Research Complex (J-PARC) was designed to meet the above requirement when necessary $[42,43]$. The equipartitioning scheme is implemented even into the recent design of an rf quadrupole linac [44].

The stability map in Fig. 4 is made up of $61 \times 61$ WARP data points obtained with initially equipartitioned waterbag beams. The abscissa is the bare phase advance $\mu_{0 \perp}$ of the single-particle betatron motion calculated together with the RFD effect. The solid and dashed lines indicate the locations of the second-order $(m=2)$ and third-order $(m=3)$ coherent resonances predicted by the conjecture in Eq. (6).

As expected, it is difficult to find a good operating area in the range $\mu_{0 \perp}>90^{\circ}$. Considerable emittance growth has also occurred along the linear synchrobetatron resonance line with $\left(n_{\perp}, n_{\|}, n^{\prime}\right)=(1,1,1)$ discussed by Hofmann and Boine-Frankenheim [40]. The linear betatron and synchrobetatron instability bands become wider at higher beam density, covering the regions $\mu_{0 \perp}>90^{\circ}$ and $\mu_{0 \perp}+\sigma_{0 \|}>$ $180^{\circ}$ almost entirely. No serious synchrotron resonance can be seen in Fig. 4, which supports the argument in Ref. [40] as long as the lattice periodicity is maintained in a sufficient level [45]. On the other hand, the excitation of weak nonlinear betatron resonances is observed below $\mu_{0 \perp}$ of $90^{\circ}$ per cell along the lines with $\left(n_{\perp}, n_{\|}, n^{\prime}\right)=(3,0,1)$ and $(4,0,1)$.

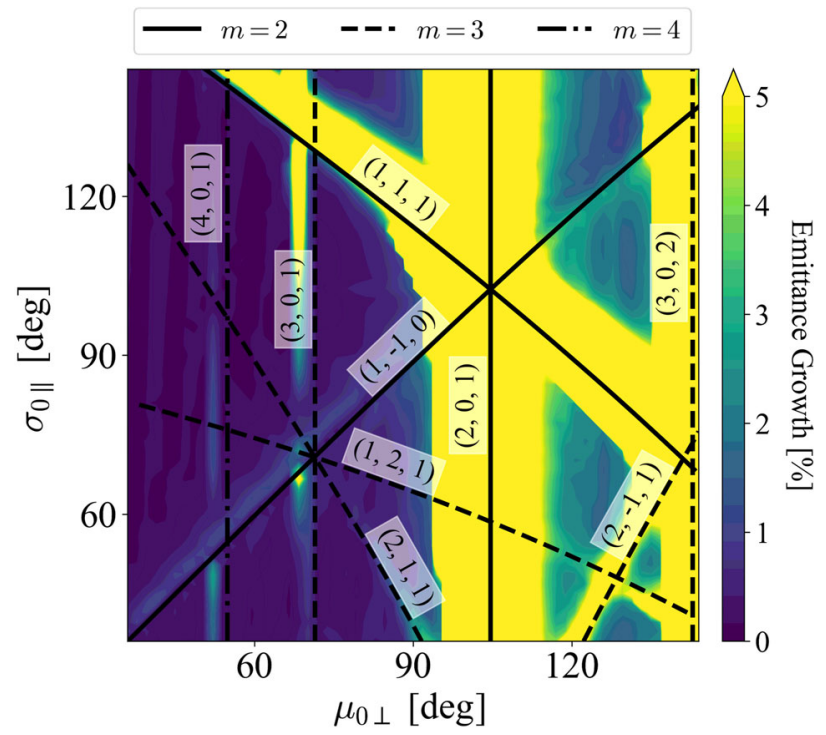

FIG. 4. WARP simulation result obtained with the equipartitioned waterbag distribution. PIC simulations were done at $3721(=61 \times 61)$ different operating points uniformly distributed in the range $36^{\circ}<\mu_{0 \perp}, \sigma_{0 \|}<144^{\circ}$, which enables us to identify instability bands wider than about $2^{\circ}$. The emittance-growth rates evaluated at the exit of the 100th AG cell are color-coded in the tune diagram. $\eta_{\perp}$ is adjusted to 0.8 over the whole tune space. The transverse emittances are also fixed at injection, while we choose the initial longitudinal emittance so as to meet the equipartitioning condition. Black lines indicate the positions of low-order coherent resonances predicted by Eq. (6) with $C_{2}=0.7$, $C_{3}=0.8$, and $C_{4}=0.9$. Three numbers beside each line represent $\left(n_{\perp}, n_{\|}, n^{\prime}\right)$.

Figure 5 shows the WARP data obtained at higher beam density. The initial parameter setting is similar to the case in Fig. 4 , but $\eta_{\perp}$ has been reduced to 0.7 . At this density, the high- $\mu_{0 \perp}$ region above $90^{\circ}$ is completely filled with wide resonance bands. A couple of betatron resonance bands are found again below $90^{\circ}$, which presumably correspond to those seen in Fig. 4 along the third-order and fourth-order coherent instability lines. These two emittance-growth bands in the waterbag beam can actually be attributed to the core instabilities of the low-order nonlinear modes. Figure 6 exhibits the transverse phase-space configurations at the exit of the 60th AG cell when the bare phase advances are set either at the point (A) or at the point (B) in Fig. 5(b). The clear deformation pattern of the beam core indicates the octupole-mode instability at the point (A) and the sextupolemode instability at the point (B). The deviations of the observed betatron resonance bands from the theoretical expectation (dashed lines), however, look significant especially in the Gaussian case, which suggests a possibility that the incoherent mechanism in the beam tail might be working. The linearization of the resonance condition with respect the rms tune shifts is also certainly an error source at such high density. Further careful investigations are needed to resolve this issue and improve the accuracy of stop-band prediction. 

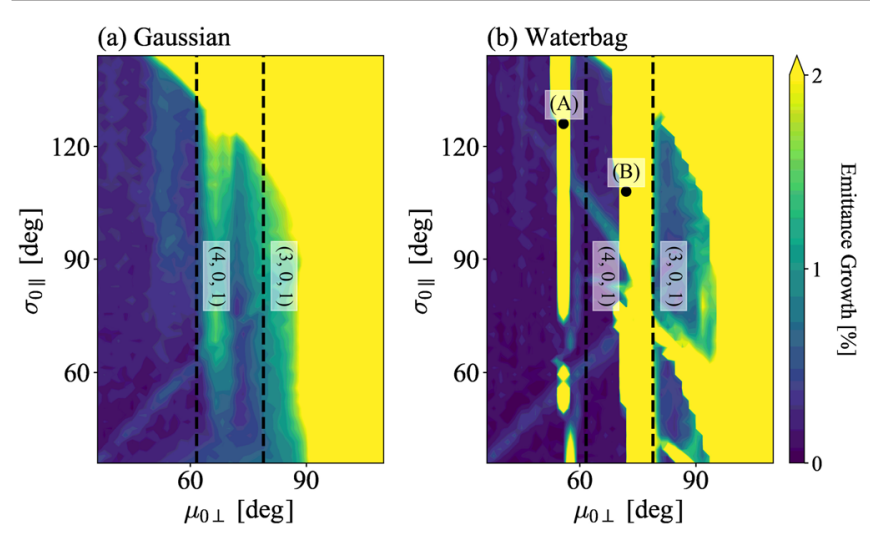

FIG. 5. WARP simulation results obtained with (a) the Gaussian beam and (b) the waterbag beam. The emittance growth is evaluated at the exit of the 100th AG cell. The initial value of $\eta_{\perp}$ is fixed at 0.7 everywhere. The transverse rms emittance $\varepsilon_{\perp}$ is also maintained in both cases, but the longitudinal parameters $\eta_{\|}$ and $\varepsilon_{\|}$are determined at each operating point so as to meet the equipartitioning condition. The locations of the noncoupling sextupole $(m=3)$ and octupole $(m=4)$ resonances expected from Eq. (6) with $C_{3}=0.8$ and $C_{4}=0.9$ are indicated by dashed lines. Three numbers in the picture represent $\left(n_{\perp}, n_{\|}, n^{\prime}\right)$. The phase-space distributions of the waterbag beam at the operating points (A) and (B) are exhibited in Fig. 6.
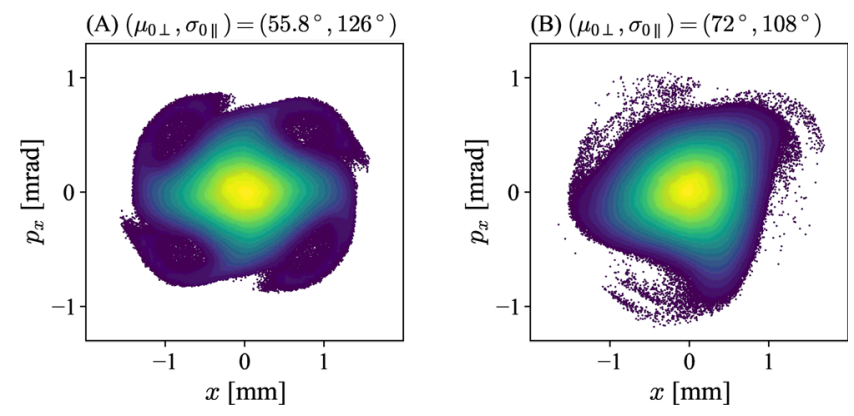

FIG. 6. Horizontal phase-space distributions after the waterbag beam goes through $60 \mathrm{AG}$ cells at the operating points (A) and (B) in Fig. 5(b). As an example, we have assumed a $10 \mathrm{MeV}$ proton bunch whose horizontal emittance is $0.1355 \pi \mathrm{mm} \cdot \operatorname{mrad}$ at injection.

\section{B. Stop bands in nonequipartitioned beams}

The equipartitioning condition in Eq. (7) is not met in many practical cases. It is thus important to ask what happens when the condition is broken. A self-consistent theory was developed by Hofmann to describe the stability properties of an anisotropic uniform-density beam propagating through a uniform focusing channel [5]. He derived the dispersion relations of various eigenmodes and concluded that beams in nonequipartitioned linac designs with medium or weak space-charge tune depression can be expected to be stable and thus not subject to emittance exchange.

WARP simulations were performed with Gaussian beams matched to the sinusoidal lattice but not equipartitioned initially. The left panel (a) in Fig. 7 shows the horizontal or vertical rms emittance growth at the exit of the 100th AG cell, whichever is greater. The longitudinal emittance growth is plotted in the right panel (b). Unlike in the previous cases of Figs. 4 and 5, the rms emittances of all three directions and the beam intensity are maintained in Fig. 7, so Eq. (7) no longer holds. Additional synchrobetatron resonances invisible in the equipartitioned case have been activated clearly. We also recognize that, under the initial condition adopted here, the emittance flows from the synchrotron motion to the betatron motion in the region $\sigma_{0 \|}>\mu_{0 \perp}$. The direction of the emittance flow is reversed in the region $\sigma_{0 \|}<\mu_{0 \perp}$.

Figure 8 shows another nonequipartitioned case where the initial rms emittances in the longitudinal and transverse directions are fixed at the values that achieve a certain ratio $\varepsilon_{\|} / \varepsilon_{\perp}$. In this simulation, the bunch intensity was determined first under the equipartitioning condition such that $\eta_{\perp}=0.7$ at the operating point $\left(\mu_{0 \perp}, \sigma_{0 \|}\right)=\left(57.6^{\circ}, 57.6^{\circ}\right)$ in the left panel and at the operating point $\left(\mu_{0 \perp}, \sigma_{0 \|}\right)=$ $\left(57.6^{\circ}, 36.0^{\circ}\right)$ in the right panel. We then adjusted the emittance ratio while keeping the intensity, and rematched the anisotropic beam to the lattice. The resultant rms tune depression corresponding to the case of Fig. 8(a) is shown in Fig. 9. We see that $\eta_{\perp}\left(\eta_{\|}\right)$tends to decrease at lower $\mu_{0 \perp}$ $\left(\sigma_{0 \|}\right)$. In the case of Fig. 8(b), $\eta_{\perp}$ varies similarly though $\eta_{\|}$ comes somewhat closer to unity, in other words, the longitudinal bunch density is a bit lower compared with the case of Fig. 8(a). Several synchrobetatron difference resonance bands are enhanced considerably in both Figs. 8(a) and 8(b) by the initial emittance imbalance that breaks the condition in Eq. (7). The primary source of the strong diagonal band is believed to be the second-order coupling term rather than the fourth-order.

According to the conjecture in Eq. (6), the instabilities of the quadrupole $(m=2)$ and sextupole $(m=3)$ modes are expected along the solid and dashed lines in Fig. 8, all of which roughly agree with the PIC observations. We suspect that particles in the Gaussian tail might be responsible for some of weak emittance-growth bands [25-27], but in any case, there seems to be no essentially new instability mechanism peculiar to an anisotropic beam. All observed instability bands should be interpreted as a consequence of regular resonances that obey a simple formula like Eq. (6).

\section{SUPPRESSION OF SYNCHROBETATRON DIFFERENCE RESONANCE}

PIC data in the last section suggests that an exchange of projected emittances between degrees of freedom takes place more seriously in a bunch with a larger emittance imbalance. The basic process seems to be simply an emittance transfer on synchrobetatron difference resonances in a periodic lattice, rather than any strong thermodynamic response toward the equipartitioned state (though 

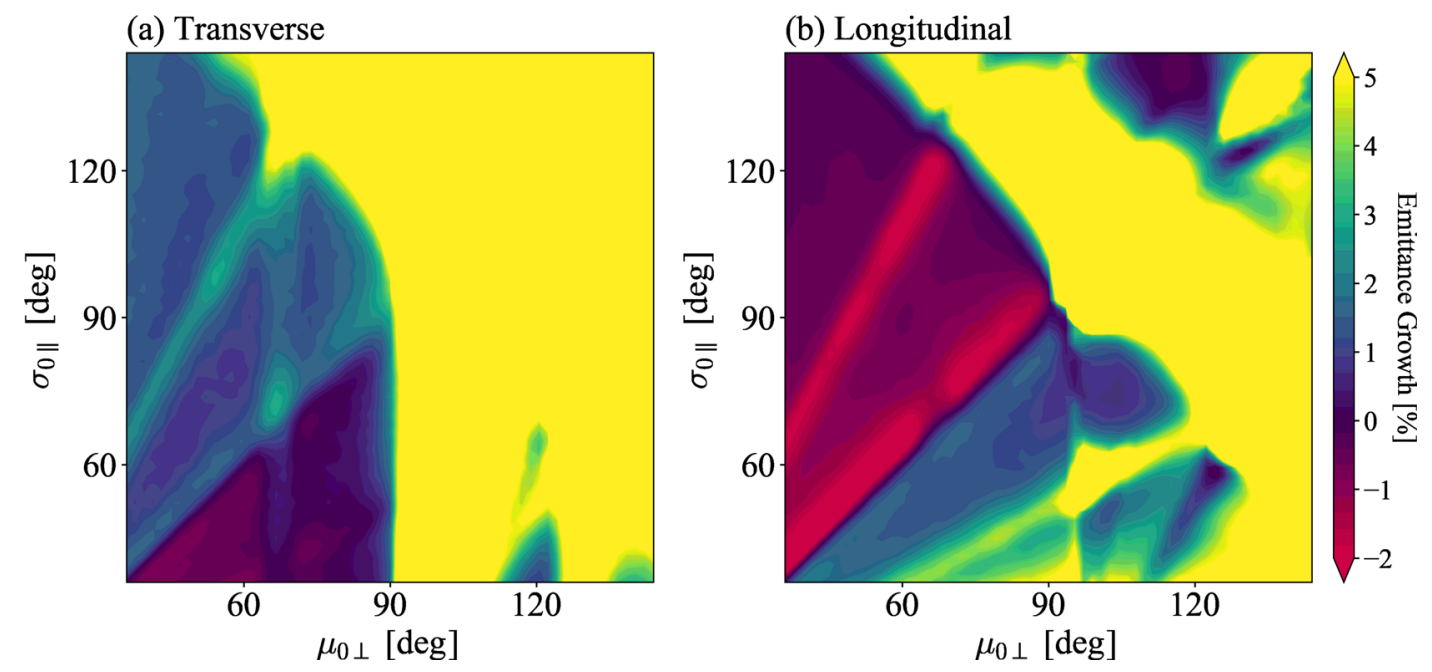

FIG. 7. WARP simulation result obtained with a nonequipartitioned Gaussian distribution. The transverse and longitudinal emittance growth rates at the exit of the 100th AG cell are plotted separately in the left panel (a) and the right panel (b). The beam intensity is fixed at the value that gives $\eta_{\perp}=0.7$ at the operating point $\left(\mu_{0 \perp}, \sigma_{0 \|}\right)=\left(57.6^{\circ}, 57.6^{\circ}\right)$.
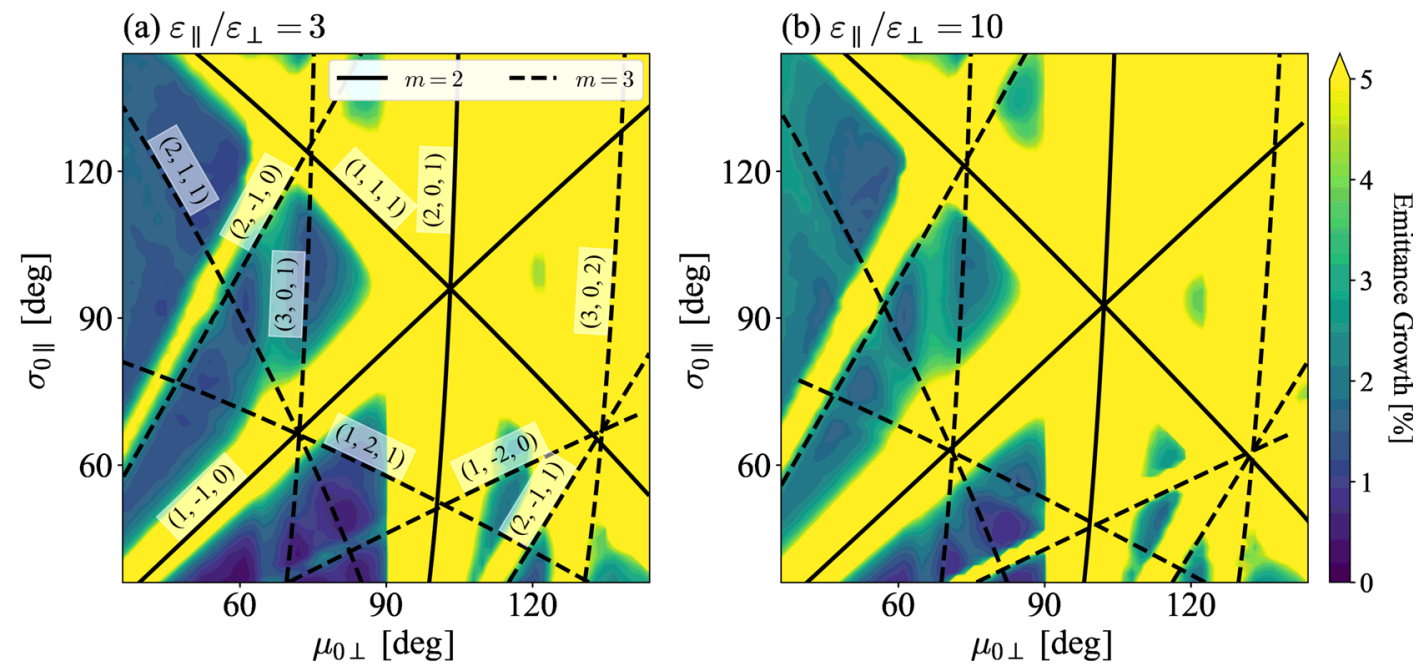

FIG. 8. WARP simulation results obtained with nonequipartitioned beams. The initial distribution is the Gaussian type that has the rms emittance ratio of (a) $\varepsilon_{\|} / \varepsilon_{\perp}=3$ and (b) $\varepsilon_{\|} / \varepsilon_{\perp}=10$. The bunch intensity and all projected emittances at injection are fixed over the whole tune space. The color is chosen based on largest emittance growth of the three directions at the exit of the 100th AG cell. The solid and dashed lines are obtained from Eq. (6) for $m=2$ and 3. Three numbers in the left picture represent $\left(n_{\perp}, n_{\|}, n^{\prime}\right)$.

some weak emittance flow was observed in Fig. 7 without resonance). The transport distance over 100 AG cells is obviously too short for an anisotropic beam to reach a state of thermodynamic equilibrium. Besides, the effect of interparticle Coulomb collisions is not incorporated in PIC simulations. The positions of the emittance-growth bands enhanced by an initial anisotropy can be predicted reasonably well by the simple resonance formula. If this anisotropy-induced instability has nothing to do with the natural thermodynamic process, we might be able to mitigate it rather easily. In the case of coasting beams, the emittance transfer between the two transverse directions can actually be suppressed under the very simple condition that $\varepsilon_{x} / \varepsilon_{y}=\left|n_{x} / n_{y}\right|$ [26], which applies to the coupling resonances driven by the term $x^{\left|n_{x}\right|} y n^{\left|n_{y}\right|}$ with $n_{x} n_{y}<0$. We anticipate that a similar argument should hold even for synchrobetatron resonances in bunched beams.

Following the previous work, we define the emittancebased quantity

$$
\Lambda\left(n_{\perp}, n_{\|}\right) \equiv \frac{\varepsilon_{\perp}}{n_{\perp}}+\frac{\varepsilon_{\|}}{n_{\|}}
$$

If our expectation is correct, the synchrobetatron difference resonance with any combination of $n_{\perp}$ and $n_{\|}\left(n_{\perp} n_{\|}<0\right)$ could be eliminated under the condition $\Lambda\left(n_{\perp}, n_{\|}\right)=0$. It does not matter whether the beam is initially 


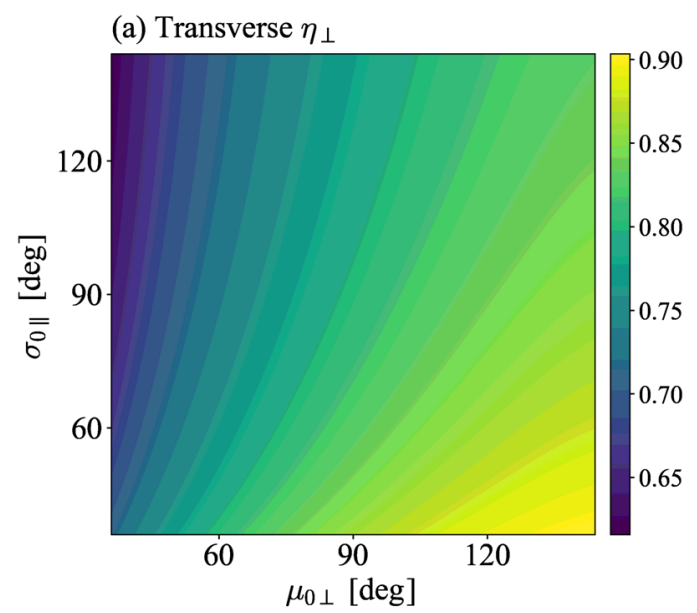

(b) Longitudinal $\eta_{\|}$

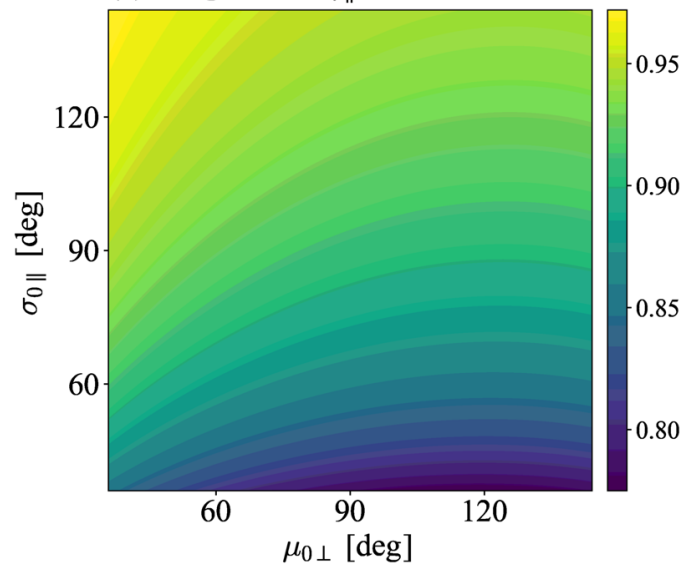

FIG. 9. Rms tune depressions in (a) the transverse direction and (b) the longitudinal direction, under the condition assumed in Fig. 8(a).

equipartitioned or anisotropic. This assertion has been partially verified by the PIC data in the last section; the difference resonance with $n_{\perp}=-n_{\|}$was always very weak when $\varepsilon_{\perp}=\varepsilon_{\|}$.

Let us try to carry out further verification, paying attention to nonlinear stop bands in Figs. 7 and 8 . Third-order synchrobetatron difference resonances are excited in both figures along the lines with $\left(n_{\perp}, n_{\|}, n^{\prime}\right)=$ $(2,-1,0)$ and $(1,-2,0)$. The condition $\Lambda\left(n_{\perp}, n_{\|}\right)=0$ leads to the emittance ratio $\varepsilon_{\|} / \varepsilon_{\perp}=0.5$ for the former resonance and $\varepsilon_{\|} / \varepsilon_{\perp}=2$ for the latter. We performed WARP simulations, adjusting the initial emittance ratio to these specific values. The coupling instability along the line $\left(n_{\perp}, n_{\|}, n^{\prime}\right)=(2,-1,0)$ is successfully suppressed in the right panel of Fig. 10 by setting $\varepsilon_{\perp}$ twice larger than $\varepsilon_{\|}$at injection. Similarly, the emittance growth along the line $\left(n_{\perp}, n_{\|}, n^{\prime}\right)=(1,-2,0)$ has disappeared in Fig. 11 under the condition $\varepsilon_{\|} / \varepsilon_{\perp}=2$. Note, however, that the linear synchrobetatron resonance with $\left(n_{\perp}, n_{\|}, n^{\prime}\right)=(1,-1,0)$
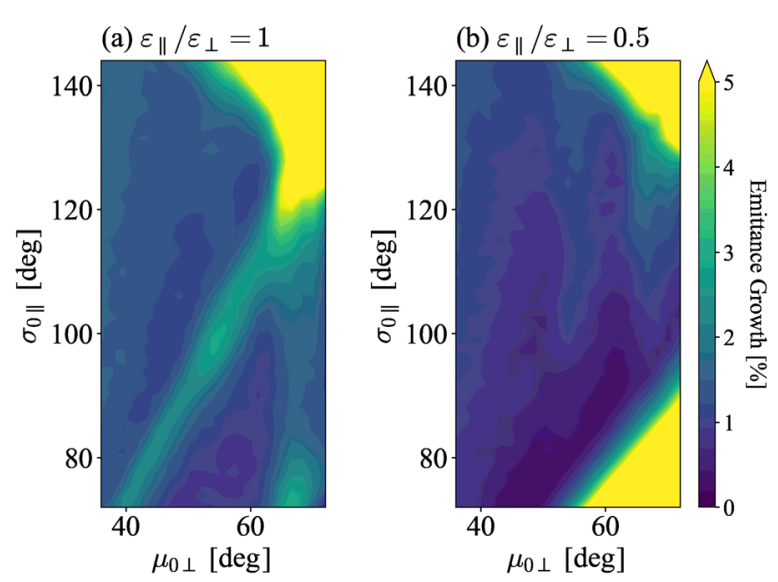

FIG. 10. WARP simulation results obtained under the initial conditions (a) $\varepsilon_{\|} / \varepsilon_{\perp}=1$ and (b) $\varepsilon_{\|} / \varepsilon_{\perp}=0.5$. The largest emittance growth of the three directions is taken to choose the color at each operating point. The result in Fig. 7 is replotted in the left panel (a) for comparison. We have changed the initial emittance ratio $\varepsilon_{\|} / \varepsilon_{\perp}$ from 1.0 to 0.5 in the right panel (b), maintaining the beam intensity.

has become serious in both cases due to the breakdown of the condition $\Lambda(1,-1)=0$ satisfied originally in Fig. 7 .

An important question is why the three major synchrobetatron difference resonances with $\left(n_{\perp}, n_{\|}, n^{\prime}\right)=$ $(1,-1,0),(2,-1,0)$ and $(1,-2,0)$, excited clearly in the nonequipartitioned cases, have vanished simultaneously in Figs. 4 and 5. This does not imply the thermodynamic enhancement of beam stability in the equipartitioned state but can readily be explained with Eq. (8). The condition $\Lambda\left(n_{\perp}, n_{\|}\right)=0$ for resonance suppression requires the emittance ratio $\varepsilon_{\|} / \varepsilon_{\perp}=-n_{\|} / n_{\perp}$. Combining this magic ratio with Eq. (7), we find

$$
n_{\perp} \eta_{\perp} \mu_{0 \perp}+n_{\|} \eta_{\|} \sigma_{0 \|}=0
$$

that lies close to the lines defined by Eq. (6) with $n^{\prime}=0$ unless the beam density is extremely high. In fact, Eq. (9) can be derived by putting $C_{m}=1$ and $n^{\prime}=0$ in Eq. (6). Since $C_{m}$ is only a bit smaller than unity for any collective modes (except for the dipole), an equipartitioned bunch automatically fulfills $\Lambda\left(n_{\perp}, n_{\|}\right) \approx 0$, which considerably weakens all synchrobetatron difference resonances with $n^{\prime}=0$. The contour plots in Fig. 12, corresponding to the case of Fig. 4, verify that $\Lambda\left(n_{\perp}, n_{\|}\right)$becomes close to zero along all three difference resonance lines mentioned above. Essentially the same resonance-suppression effect under the equipartitioning condition has been confirmed for coasting beams as well [26,27].

\section{CONCLUDING REMARKS}

We have conducted a comprehensive numerical study of resonant instability in an intense hadron bunch exposed to 

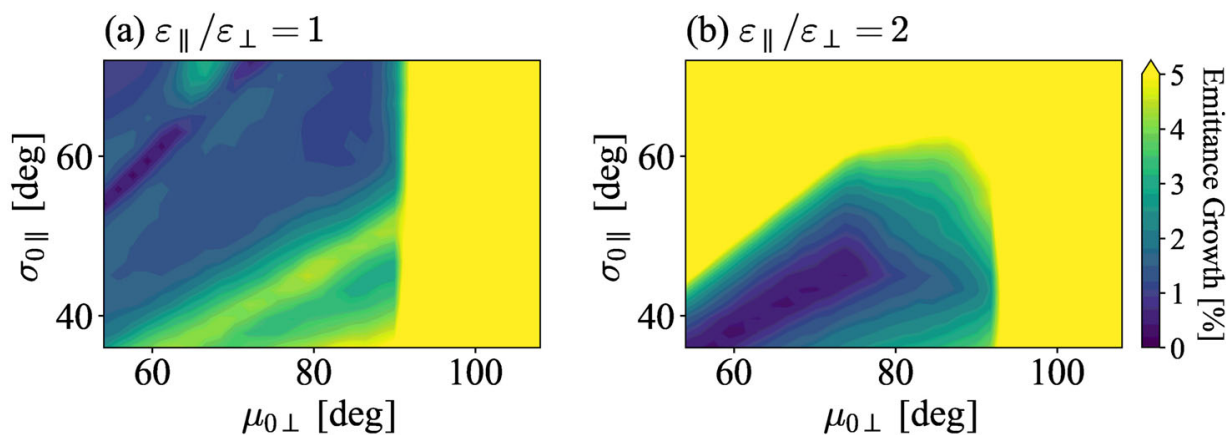

FIG. 11. WARP simulation results obtained with the initial emittance ratios (a) $\varepsilon_{\|} / \varepsilon_{\perp}=1$ and (b) $\varepsilon_{\|} / \varepsilon_{\perp}=2$. The simulation conditions are the same as in Fig. 10 except for the value of $\varepsilon_{\|} / \varepsilon_{\perp}$ in the right panel.
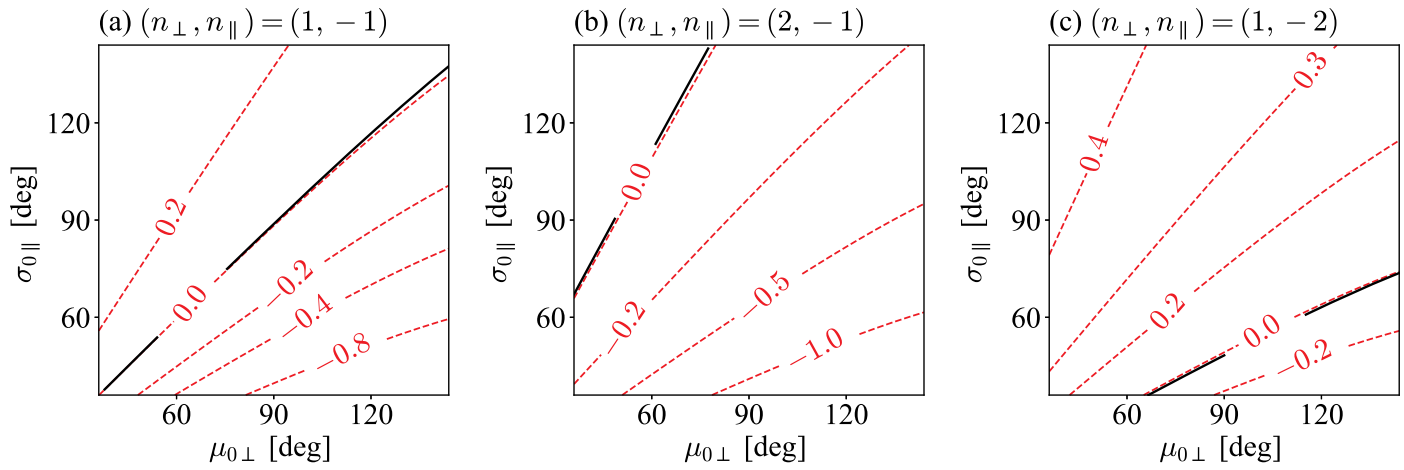

FIG. 12. Contour plots of $\Lambda\left(n_{\perp}, n_{\|}\right)$in an equipartitioned case. The same initial beam conditions as considered in Fig. 4 are taken to calculate the $\Lambda$-value at each operating point with (a) $\left(n_{\perp}, n_{\|}, n^{\prime}\right)=(1,-1,0)$, (b) $\left(n_{\perp}, n_{\|}, n^{\prime}\right)=(2,-1,0)$, and (c) $\left(n_{\perp}, n_{\|}, n^{\prime}\right)=$ $(1,-2,0)$. A number on each contour line is the value of $\Lambda\left(n_{\perp}, n_{\|}\right)$divided by a normalization constant, i.e., the value of $\Lambda(1,1)$ at the operating point $\left(\mu_{0 \perp}, \sigma_{0 \|}\right)=\left(60^{\circ}, 60^{\circ}\right)$. Black solid lines are obtained from Eq. (6) with $C_{2}=0.7$ or $C_{3}=0.8$.

periodic focusing forces in all three degrees of freedom. The period of the AG potential in the transverse plane was set twice longer than the longitudinal focusing period, assuming a typical DTL situation. The simple sinusoidal waveform was taken to model the standard FODO quadrupole lattice. The longitudinal weak focusing effect from rf accelerating gaps and resultant transverse RFD were also incorporated on the basis of the equivalent sinusoidal model. As these external driving forces have been linearized, all nonlinear instabilities revealed in the WARP simulations here are caused solely by the space-charge potential.

The excitation of various nonlinear resonances was observed in both Gaussian and waterbag bunches, depending on the initial beam conditions. While the pure synchrotron resonance with $n_{\perp}=0$ did not appear seriously, low-order betatron and synchrobetatron resonances turned out to be problematic especially in a nonequipartitioned bunch. We have confirmed in separate simulations that nonlinear coupling resonances are enhanced in mismatched beams as well. These space-charge-driven instability bands are distributed even below the bare betatron and synchrotron phase advances of $90^{\circ}$ per cell, limiting the usable operating area in the tune diagram. In the range $\mu_{0 \perp}>90^{\circ}$, it is almost hopeless to find a sufficiently wide resonance-free area at high-bunch density, which is consistent with the conventional design criterion.

The 3D version of the coherent resonance conjecture in Eq. (6) appears to be useful for predicting approximate locations of stop bands, provided that the rms tune depression is not too far from unity. We could hence search for optimum operating parameters of a high-intensity linac in a similar way as recently proposed for the conceptual design of a circular lattice [25-27]. It seems possible to establish a universal picture of resonances in a matched beam, no matter whether the machine is linear or circular [46].

The emittance exchange between the transverse and longitudinal directions on a specific synchrobetatron difference resonance can be made inactive by imposing the condition $\Lambda\left(n_{\perp}, n_{\|}\right)=0$ on the initial emittance ratio. This is a natural generalization of the previously discovered condition for the suppression of difference resonances in a coasting beam [26]. As proved in Sec. VI, there exists the magic emittance ratio that deactivates a particular synchrobetatron resonance. Choosing such parameters as to meet the condition in Eq. (7) is found to be advantageous because that choice automatically makes $\Lambda\left(n_{\perp}, n_{\|}\right)$close to zero along a few low-order synchrobetatron difference resonance lines. 


\section{ACKNOWLEDGMENTS}

The authors would like to thank C. Ichikawa for the WARP simulation data in Appendix B. They are also indebted to Dr. Y. Liu for information about the J-PARC DTL. This work is supported partially by JSPS KAKENHI Grant No. 18H03472. A part of the numerical results in this paper was obtained with the computer resource offered by Research Institute for Information Technology, Kyushu University.

\section{APPENDIX A: PHASE-SPACE MATCHING OF AN INITIAL PARTICLE DISTRIBUTION AT HIGH SPACE-CHARGE DENSITY}

It is extremely important to construct an initial particle distribution three-dimensionally matched to the AG lattice as precisely as possible. In the old days, the concept of the rms matching was often employed to generate an initial distribution in the presence of space-charge interaction, but such a crude matching procedure certainly fails at highbeam density. Since the Coulomb self-field potential is nonlinear, not only the second moments but also higher order moments have to be adapted carefully to the lattice; in other words, it is necessary to provide a phase-space distribution corresponding to the stationary solution to the Vlasov-Poisson equation system. As is well known, the beam profile in real space is more and more homogenized due to the Debye screening effect as the beam becomes denser in phase space. A serious collective oscillation is inevitably excited in the core of an rms matched beam at high density, leading to an emittance blowup and resultant halo formation [47-49]. This unwanted natural effect makes it troublesome to identify the signatures of weak resonances in PIC simulations.

It is possible to define a variety of stationary distribution functions when the external focusing force is uniform. In the case of AG focusing, however, a sophisticated matching procedure is required to establish a stationary state adapted to the periodic lattice. Lund and his co-workers developed an efficient numerical technique for the construction of 2D particle distributions well matched to an arbitrary AG lattice at high space-charge density [50]. We generalized their 2D prescription to 3D for the present simulation study of bunched-beam stability.

The outline of the 3D matching procedure is the following. As the first step, a spherical stationary state is constructed based on the $s$-independent (smoothed) Hamiltonian of the form

$$
\bar{H}_{b}=\frac{p_{x}^{2}+p_{y}^{2}+p_{z}^{2}}{2}+\frac{1}{2} \kappa_{b} r^{2}+I_{q} \phi_{\mathrm{sc}}(r),
$$

where $r=\sqrt{x^{2}+y^{2}+z^{2}}$. Considering the 3D rms envelope equation, the coefficient $\kappa_{b}$ of the linear focusing term is defined as

$$
\kappa_{b}=\frac{3 \lambda_{3} \Gamma}{2 a_{b}^{3}} \int_{0}^{\infty} \frac{d \zeta}{(1+\zeta)^{5 / 2}}+\frac{\varepsilon_{b}^{2}}{a_{b}^{4}},
$$

where $\varepsilon_{b}$ is a sort of average initial rms emittance given by $\varepsilon_{b}=\left(\varepsilon_{x} \varepsilon_{y} \varepsilon_{z}\right)^{1 / 3}$ and $a_{b}=\left(a_{x} a_{y} a_{z}\right)^{1 / 3}$ with $a_{w}(w=x, y, z)$ being the stationary solution to Eq. (2). Although $a_{b}$ varies periodically along the beam line, the variation is generally quite small under a typical operating condition. In Eq. (A2), therefore, we use the value of $a_{b}$ at the entrance $(s=0)$ to evaluate $\kappa_{b}$. We then try to find a stationary distribution in this smoothed system. Since $\bar{H}_{b}$ is a constant of motion, an arbitrary function of $\bar{H}_{b}$, i.e., $f\left(\bar{H}_{b}\right)$ satisfies the Vlasov equation. The scalar potential $\phi_{\mathrm{sc}}$ of the stationary beam can be derived from the Poisson equation

$$
\frac{1}{r^{2}} \frac{d}{d r}\left(r^{2} \frac{d \phi_{\mathrm{sc}}}{d r}\right)=-\frac{q}{\epsilon_{0}} \iiint f\left(\bar{H}_{b}\right) d p_{x} d p_{y} d p_{z},
$$

where $q$ is the charge state of the particle and $\epsilon_{0}$ the permittivity of vacuum. Finally, the spherical distribution $f\left(\bar{H}_{b}\right)$ is adapted to the AG lattice by transforming the canonical variables of individual particles at $s=0$ as

$$
\begin{aligned}
w & \rightarrow\left(\frac{a_{w}}{a_{b}}\right)_{s=0} w \\
p_{w} & \rightarrow\left(\frac{a_{b} \varepsilon_{w}}{a_{w} \varepsilon_{b}}\right)_{s=0} p_{w}+\left(\frac{1}{a_{b}} \frac{d a_{w}}{d s}\right)_{s=0} w .
\end{aligned}
$$

The pseudoequilibrium distribution constructed in this way does not exactly reflect the self-consistent solution to the Vlasov-Poisson equation system, but the matching is remarkably well in the cases considered in the present study. We, however, suspect that the matching error may be enhanced under stronger focusing and/or in a bunch with the aspect ratio far from unity.

Figure 13 displays an example of rms emittance evolutions of the Gaussian bunches generated by the quasiselfconsistent 3D matching procedure (black line) and by the classical rms matching procedure (red line). As expected, the rms matched beam has experienced a significant emittance jump right after the injection. In contrast, almost no emittance growth has occurred in the beam produced by our new stationary distribution generator.

The initial and final particle distributions in the horizontal phase space are depicted in Fig. 14 for reference. The pseudoequilibrium initial distribution in the upper left panel, provided by the matching algorithm outline above, is obviously different from the rms matched Gaussian in the lower left. The former has a rectangularlike shape as a result of the Debye screening effect. The latter has an elliptical profile at the beginning but is eventually redistributed into the rectangular configuration analogous to the pseudoequilibrium beam. 


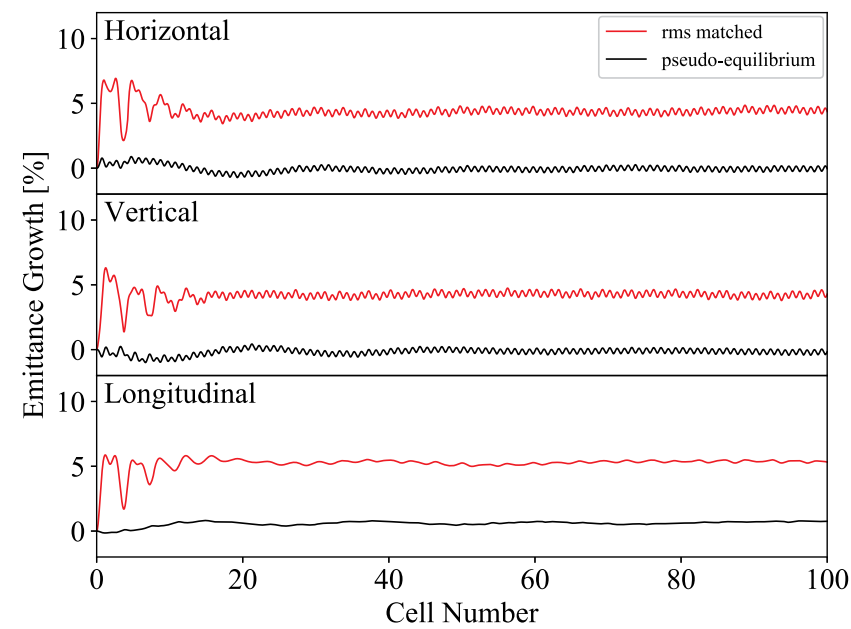

FIG. 13. Time evolution of the projected rms emittances of a $10 \mathrm{MeV}$ proton beam at the operating point $\left(\mu_{0 \perp}, \sigma_{0 \|}\right)=$ $\left(60^{\circ}, 60^{\circ}\right)$. The beam initially has the equipartitioned Gaussian distribution whose transverse tune depression is adjusted to $\eta_{\perp}=0.6$. Black curves correspond to the case where the phase-space configuration is in the pseudoequilibrium, while red curves to the case of rms matching.

(a) Pseudo-equilibrium
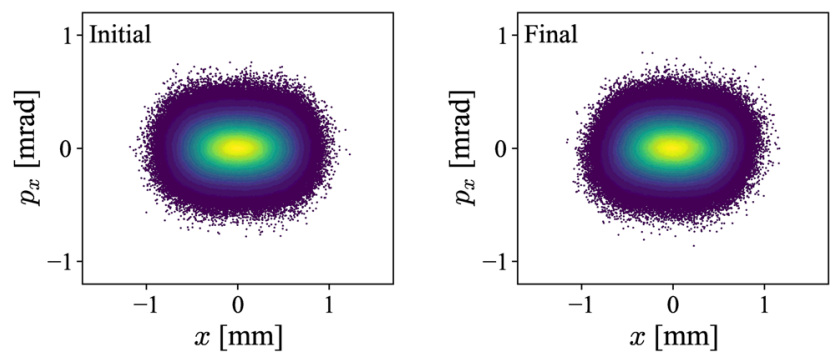

(b) RMS matched
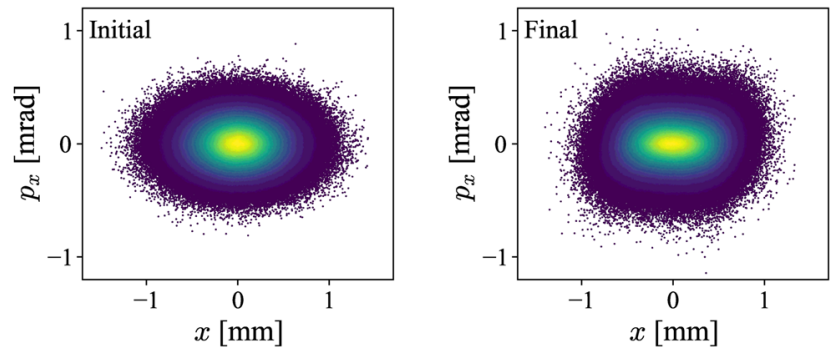

FIG. 14. Initial and final particle distributions in the horizontal phase space, corresponding to the WARP simulations in Fig. 13. The transverse rms emittance has been assumed initially to be about $0.05 \pi \mathrm{mm} \cdot \mathrm{mrad}$. In the upper panel (a), the beam is initially in a pseudoequilibrium state while in the lower panel (b), rms matched to the lattice at injection.

\section{APPENDIX B: BETATRON STABILITY MAP WITH A FIXED SYNCHROTRON PHASE ADVANCE}

The 3D coherent resonance conjecture applicable to the case where $\mu_{0 x} \neq \mu_{0 y}$ may be written as

$$
\begin{aligned}
& n_{x} \mu_{0 x}\left[1-C_{m}\left(1-\eta_{x}\right)\right]+n_{y} \mu_{0 y}\left[1-C_{m}\left(1-\eta_{y}\right)\right] \\
& +n_{\|} \sigma_{0 \|}\left[1-C_{m}\left(1-\eta_{\|}\right)\right]=\pi n^{\prime} .
\end{aligned}
$$

This condition predicts many stop bands, but needless to say, all of them are not equally important in practice. Some might be almost invisible just like the pure synchrotron resonance. It depends on several factors such as the initial emittance condition, lattice design, transport distance, and even phase-space configuration of the beam. Providing a reliable guideline to judge resonances of high practical importance is a future issue. We would, however, say that it is most advisable to pay attention to all coherent stop bands of up to the third order for safety in the conceptual design stage of a high-intensity linac. This automatically covers space-charge-driven incoherent tail resonances of up to the sixth order.

An example of the betatron stability map obtained with a fixed synchrotron phase advance is exhibited in Fig. 15. The solid lines indicate the positions of the linear $(m=2)$ resonances predicted by Eq. (B1). The difference resonance $k \mu_{0 x}-k \mu_{0 y}=0 \quad(k=$ integer $)$ is completely suppressed because $\varepsilon_{x}=\varepsilon_{y}$ along the line. Two linear synchrobetatron resonance bands with $\left(n_{x}, n_{y}, n_{\|}, n^{\prime}\right)=(-1,0,1,0)$ and $(0,-1,1,0)$ also become negligible because the condition $\Lambda\left(n_{\perp}, n_{\|}\right)=0$ is fulfilled along them in this simulation. Other relatively weak instabilities recognizable in the map can be interpreted as third-order coherent resonances with

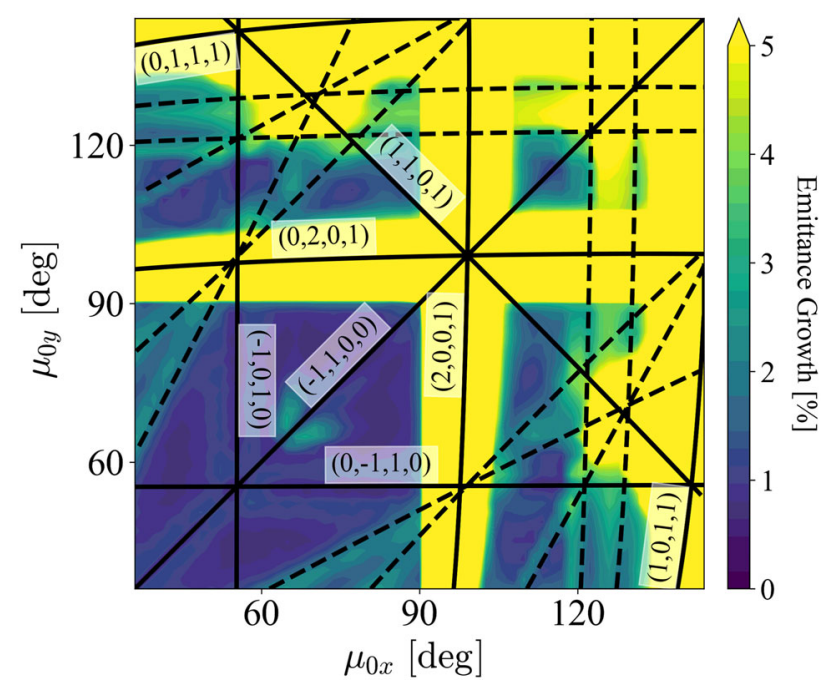

FIG. 15. WARP simulation results with the bare synchrotron tune $\sigma_{0 \|}$ fixed at $55^{\circ}$. The initial phase-space distribution of particles in the bunch is the Gaussian type. The projected rms emittances in the three spatial directions are equalized everywhere in the diagram. The beam intensity is maintained at the value that gives $\eta_{\perp}=0.8$ at the operating point $\left(\mu_{0 \perp}, \sigma_{0 \|}\right)=$ $\left(60^{\circ}, 60^{\circ}\right)$. Solid and dashed lines in the picture indicate the locations of the possible second-order and third-order stop bands obtained from Eq. (B1) with $C_{2}=0.7$ and $C_{3}=0.8$. Four integers written beside each line represent $\left(n_{x}, n_{y}, n_{\|}, n^{\prime}\right)$. 
$\left(n_{x}, n_{y}, n_{\|}, n^{\prime}\right)=(0,3,0,2),(3,0,0,2),(0,2,-1,1),(2,0$, $-1,1),(-1,2,0,1),(2,-1,0,1),(2,-1,0,0),(-1,2,0,0)$, $(1,-1,1,0)$, and $(1,-1,-1,0)$ or as incoherent tail resonances of twice the order. Dashed lines in the picture indicate the positions of these nonlinear resonances expected from Eq. (B1). Some of the third-order synchrobetatron resonance lines, not drawn here, have overlapped with the linear betatron resonances with $\left(n_{x}, n_{y}, n_{\|}, n^{\prime}\right)=$ $(2,0,0,1)$ and $(0,2,0,1)$.

[1] M. Reiser, Theory and Design of Charged Particle Beams (John Wiley \& Sons, New York, 2008).

[2] T. Wangler, RF Linear Accelerators (John Wiley \& Sons, New York, 1998).

[3] R. L. Gluckstern, Oscillation modes in two-dimensional beams, in Proceedings of the 1970 Linear Accelerator Conference, edited by M. R. Tracy (Fermilab, Batavia, Illinois, 1970), p. 811.

[4] I. Hofmann, L. J. Laslett, L. Smith, and I. Habor, Stability of the Kapchinskij-Vladimirskij (K-V) distribution in long periodic transport systems, Part. Accel. 13, 145 (1983).

[5] I. Hofmann, Stability of anisotropic beams with space charge, Phys. Rev. E 57, 4713 (1998).

[6] H. Okamoto and K. Yokoya, Parametric resonances in intense one-dimensional beams propagating through a periodic focusing channel, Nucl. Instrum. Methods Phys. Res., Sect. A 482, 51 (2002).

[7] I. Hofmann, G. Franchetti, O. Boine-Frankenheim, J. Qiang, and R. D. Ryne, Space charge resonances in two and three dimensional anisotropic beams, Phys. Rev. ST Accel. Beams 6, 024202 (2003).

[8] L. Groening, I. Hofmann, W. Barth, W. Bayer, G. Clemente, L. Dahl, P. Forck, P. Gerhard, M. S. Kaiser, M. Maier, S. Mickat, T. Milosic, S. Yaramyshev, and D. Uriot, Experimental Evidence of Space Charge Driven Emittance Coupling in High Intensity Linear Accelerators, Phys. Rev. Lett. 103, 224801 (2009).

[9] L. Groening, W. Barth, W. Bayer, G. Clemente, L. Dahl, P. Forck, P. Gerhard, I. Hofmann, M. S. Kaiser, M. Maier, S. Mickat, T. Milosic, D. Jeon, and D. Uriot, Experimental Evidence of the $90^{\circ}$ Stop Band in the GSI UNILAC, Phys. Rev. Lett. 102, 234801 (2009).

[10] D.-O. Jeon, Experimental evidence of space charge driven resonances in high intensity linear accelerators, Phys. Rev. Accel. Beams 19, 010101 (2016).

[11] J. Qiang, R. D. Ryne, S. Habib, and V. Decyk, An objectoriented parallel particle-in-cell code for beam dynamics simulation in linear accelerators, J. Comput. Phys. 163, 434 (2000).

[12] D. P. Grote, A. Friedman, G. D. Craig, I. Haber, and W. M. Sharp, Progress toward source-to-target simulation, Nucl. Instrum. Methods Phys. Res., Sect. A 464, 563 (2001).

[13] H. Wiedemann, Particle Accelerator Physics (SpringerVerlag, Berlin, 1993).

[14] H. Okamoto, K. Kojima, and K. Ito, A compact Paul ion trap for the study of space-charge effects in drift-tube linear accelerators, Prog. Theor. Exp. Phys. 2019, 093G01 (2019).

[15] M. L. Good, Phase reversal focusing in linear accelerator, Phys. Rev. 92, 538 (1953).

[16] Ia. B. Fainberg, Alternating phase focusing, Technical Report No. CERN-91-100, CERN, Geneva, 1956, p. 91.

[17] W.-H. Cheng, R. L. Gluckstern, and H. Okamoto, Synchrobetatron-coupling effects in alternating-phasefocusing linacs, Phys. Rev. E 48, 4689 (1993).

[18] K. Fukushima, K. Ito, H. Okamoto, S. Yamaguchi, K. Moriya, H. Higaki, T. Okano, and S. M. Lund, Experimental verification of resonance instability bands in quadrupole doublet focusing channels, Nucl. Instrum. Methods Phys. Res., Sect. A 733, 18 (2014).

[19] K. Ito, H. Okamoto, Y. Tokashiki, and K. Fukushima, Coherent resonance stop bands in alternating gradient beam transport, Phys. Rev. Accel. Beams 20, 064201 (2017).

[20] H. Okamoto, Y. Wada, and R. Takai, Radio-frequency quadrupole trap as a tool for experimental beam physics, Nucl. Instrum. Methods Phys. Res., Sect. A 485, 244 (2002).

[21] R. Takai, H. Enokizono, K. Ito, Y. Mizuno, K. Okabe, and H. Okamoto, Development of a compact plasma trap for experimental beam physics, Jpn. J. Appl. Phys. 45, 5332 (2006).

[22] F. J. Sacherer, RMS envelope equations with space charge, IEEE Trans. Nucl. Sci. 18, 1105 (1971).

[23] W. Montague, Fourth-order coupling resonances excited by space-charge forces in a synchrotron, Technical Report No. CERN/ISR/68-38, CERN, Geneva, 1968.

[24] The beam injection procedure is usually much more complex in circular machines than in linacs. Even if the condition $\varepsilon_{x} / \varepsilon_{y}=1$ is eventually met in a ring, the difference resonance could deteriorate the beam quality unless the two emittances are kept balanced throughout the accumulation process.

[25] H. Okamoto, M. Aoki, C. Ichikawa, K. Kojima, T. Kurauchi, and Y. Yamane, Coherent and incoherent space-charge effects in high-intensity hadron rings, J. Instrum. 15, P07017 (2020).

[26] K. Kojima, H. Okamoto, and Y. Tokashiki, Empirical condition of betatron resonances with space charge, Phys. Rev. Accel. Beams 22, 074201 (2019).

[27] K. Kojima, H. Okamoto, and Y. Tokashiki, Reply to "Comment on 'Empirical condition of betatron resonances with space charge"”, Phys. Rev. Accel. Beams 23, 028002 (2020).

[28] F. J. Sacherer, Transverse space-charge effects in circular accelerators, Ph.D thesis, Lawrence Radiation Laboratory, USA, 1968 [Technical Report No. UCRL-18454, 1968].

[29] S. Machida, Space-charge effects in low-energy proton synchrotrons, Nucl. Instrum. Methods Phys. Res., Sect. A 309, 43 (1991).

[30] R. Baartman, Betatron resonances with space charge, AIP Conf. Proc. 448, 56 (1998).

[31] Y. Tokashiki, K. Fukushima, and H. Okamoto, Twodimensional Vlasov analysis of collective resonances in intense charged-particle beams, in Proceedings of the 71st Spring Meeting of the Japanese Physical Society, 2016, 19aAQ-1 (The Physical Society of Japan, 2016), p. 553, ISSN 2189-079X. 
[32] Y. Tokashiki, Theoretical study of collective resonance instability in high-intensity beams, Master thesis, AdSM, Hiroshima University, 2017.

[33] C. Li and R. A. Jameson, Structure resonances due to space charge in periodic focusing channels, Phys. Rev. Accel. Beams 21, 024204 (2018).

[34] J. Struckmeier and M. Reiser, Theoretical studies of envelope oscillations and its instabilities of mismatched intense charged-particle beams in periodic focusing channels, Part. Accel. 14, 227 (1984).

[35] J. Qiang, Three-dimensional envelope instability in periodic focusing channel, Phys. Rev. Accel. Beams 21, 034201 (2018).

[36] C. Chen and R. C. Davidson, Nonlinear Resonances and Chaotic Behavior in a Periodically Focused Intense Charged-Particle Beams, Phys. Rev. Lett. 72, 2195 (1994).

[37] S. Y. Lee and A. Riabko, Envelope Hamiltonian of an intense charged-particle beam in periodic solenoidal fields, Phys. Rev. E 51, 1609 (1995).

[38] R. Pakter and F. B. Rizzato, Stability of Periodically Focused Intense Particle Beams, Phys. Rev. Lett. 87, 044801 (2001).

[39] S. M. Lund and B. Bukh, Stability properties of the transverse envelope equations describing intense ion beam transport, Phys. Rev. ST Accel. Beams 7, 024801 (2004).

[40] I. Hofmann and O. Boine-Frankenheim, Revising the Longitudinal $90^{\circ}$ Limit in High Intensity Linear Accelerators, Phys. Rev. Lett. 118, 114803 (2017).

[41] R. A. Jameson, Beam-intensity limitations in linear accelerators, IEEE Trans. Nucl. Sci. 28, 2408 (1981).

[42] M. Ikegami, Beam commissioning and operation of the J-PARC linac, Prog. Theor. Exp. Phys. 2012, $02 B 002$ (2012).
[43] Y. Liu et al., Progress of J-PARC linac commissioning, in Proceedings of the 10th International Particle Accelerator Conference (IPAC2019) (Melbourne, Australia, 2019), p. 1990.

[44] Y. Kondo, T. Morishita, and R. A. Jameson, Development of a radio frequency quadrupole linac implemented with the equipartitioning beam dynamics scheme, Phys. Rev. Accel. Beams 22, 120101 (2019).

[45] A. Rubin, L. Groening, and I. Hofmann, Simulation study of a high-intensity linear accelerator operated at longitudinal phase advances above $90^{\circ}$, Phys. Rev. Accel. Beams 23, 124202 (2020).

[46] One must be careful not to confuse the fundamental resonance issue of a matched beam with what happens in a beam far from the ideal stationary state. Complex transient phenomena during, e.g., the beam injection and accumulation processes in a ring are beyond the scope of perturbative resonance theories.

[47] J. Struckmeier, J. Klabunde, and M. Reiser, On the stability and emittance growth of different particle phase-space distributions in a long magnetic quadrupole channel, Part. Accel. 15, 47 (1984).

[48] H. Okamoto and M. Ikegami, Simulation study of halo formation in breathing round beams, Phys. Rev. E 55, 4694 (1997).

[49] A. V. Fedotov, R. L. Gluckstern, S. S. Kurennoy, and R. D. Ryne, Halo formation in three-dimensional bunches with various phase space distributions, Phys. Rev. ST Accel. Beams 2, 014201 (1999).

[50] S. M. Lund, T. Kikuchi, and R. C. Davidson, Generation of initial kinetic distributions for simulation of long-pulse charged particle beams with high space-charge intensity, Phys. Rev. ST Accel. Beams 12, 114801 (2009). 